\title{
FRACTIONAL INTEGRAL FORMULAE CONCERNING CERTAIN SPECIAL FUNCTIONS
}

\author{
V.B.L. CHAURASIA AND HARI SINGH PARIHAR
}

\begin{abstract}
The main aim of the present paper is to derive some results related to fractional integral formulae on the product of multivariable $H$-function and two general class of multivariable polynomials. A large number of known and new result have also been obtained by proper choice of parameters.
\end{abstract}

\section{Introduction}

From the last four decades several mathematician (see, for example Ross [2], Kilbas and Saigo [1], Srivastava and Goyal [12], Srivastava and Hussain [6], Srivastava, Chandel and Vishwakarma 10], Manocha and Sharma [3], Saigo and Raina 17], Dhami and Gaira [15], Lin, Tu and Srivastava 19], Oldham and Spanier [13], Chaurasia and Godika 21], and Chaurasia and Gupta [20]) have made great and significant contribution in the field of fractional calculus, specially fractional derivatives and fractional integrals involving various functions.

The computation of fractional integrals (and fractional derivatives) of transcendental function of one and more variables are important from the point of view of the usefulness of these results in (for example) the evaluation of differential and integral equation. Motivated by these and other avenues of applications, a several mathematician and physician have made use of the fractional calculus operator in the theory of special functions of one and more variables.

In this paper, we are obtaining some results by using fractional integral operators on the product of general class of multivariable polymonials and multivariable $H$-function. Ross, B. [2] defined the fractional integral operator as the special case of the RiemannLiouville fractional integral operator for $c=0$ and represented it as ${ }_{c} I_{x}^{v}\{f(x)\}$.

Received December 29, 2005; revised June 28, 2006.

2000 Mathematics Subject Classification. 33C70, 26A33.

Key words and phrases. Fractional integral formulae, a general class of multivariable polynomials, multivariable $H$-function, generalized Lauricella function. 
The $H$-function of several complex variables [1] is defined in the following form:

$$
\begin{aligned}
& H\left[x_{1}, \ldots, x_{r}\right] \\
& =H_{P, Q:\left(P^{\prime}, Q^{\prime}\right) ; \ldots ;\left(P^{(r)}, Q^{(r)}\right)}^{0, N:\left(M^{\prime} N^{\prime}\right) ; \ldots ;\left(M^{(r)}, N^{(r)}\right)}\left[\begin{array}{l}
{\left[(a): \theta^{\prime}, \ldots, \theta^{(r)}\right]:\left[\left(b^{\prime}\right): \phi^{\prime}\right] ; \cdots ;\left[\left(b^{(r)}\right): \phi^{(r)}\right] ;} \\
{\left[(c): \psi^{\prime}, \ldots, \psi^{(r)}\right]:\left[\left(d^{\prime}\right): \delta^{\prime}\right] ; \cdots ;\left[\left(d^{(r)}\right): \delta^{(r)}\right] ;}
\end{array} x_{1}, \ldots, x_{r}\right] \\
& =\frac{1}{(2 \pi \omega)^{r}} \int_{L_{1}} \cdots \int_{L_{r}} m_{1}\left(\xi_{1}\right) \cdots m_{r}\left(\xi_{r}\right) \eta\left(\xi_{1}, \ldots, \xi_{r}\right) x_{1}^{\xi_{1}} \cdots x_{r}^{\xi_{r}} d \xi_{1} \cdots d \xi_{r}, \\
& \quad \text { where } \omega=\sqrt{-1} .
\end{aligned}
$$

The convergence conditions and other details of the above function are given by Srivastava et al. 5, p.251, eq.(C.1)], see also equations (1.3) and (1.4) below

$$
\begin{aligned}
& \Lambda_{i}=\sum_{j=N+1}^{P} \theta_{j}^{(i)}-\sum_{j=1}^{Q} \psi_{j}^{(i)}+\sum_{j=1}^{N^{(i)}} \phi_{j}^{(i)}-\sum_{j=N^{(i)}+1}^{P^{(i)}} \phi_{j}^{(i)}+\sum_{j=1}^{M^{(i)}} \delta_{j}^{(i)}-\sum_{j=M^{(i)}+1}^{Q^{(i)}} \delta_{j}^{(i)}>0 \\
& \alpha_{i}=\min \left\{\operatorname{Re}\left(d_{j}^{(i)} / \delta_{j}^{(i)}\right\}, \quad j=1, \ldots, M^{(i)}, \quad \forall i \in(1, \ldots, r)\right.
\end{aligned}
$$

We assume that the convergence and existence conditions of above function are satisfied by each of the various $H$-function involved throuthout the present work.

A general class of multivariable polynomials [8, p.686, eq.(1.4)], defined as follows

$$
S_{n}^{m_{1}, \ldots, m_{s}}\left[w_{1} \cdots w_{s}\right]=\sum_{k_{1}, \ldots, k_{s}=0}^{m_{1} k_{1}+\cdots+m_{s} k_{s} \leq n}(-n)_{m_{1} k_{1}+\cdots+m_{s} k_{s}} A\left(n ; k_{1}, \ldots, k_{s}\right) \frac{\left(w_{1}\right)^{k_{1}}}{k_{1} !} \cdots \frac{\left(w_{s}\right)^{k_{s}}}{k_{s} !}
$$

where $m_{1}, \ldots, m_{s}$ are arbitrary positive integers and the coefficients $A\left(n ; k_{1} \cdots k_{s}\right),\left(n ; k_{i^{\prime}}\right.$ $\left.\geq 0, i^{\prime}=1, \ldots, s\right)$ are arbitrary constants, real or complex.

For the sake of brevity, we use here the following notations

$$
\begin{aligned}
A(\phi)= & \sum_{k_{1}, \ldots, k_{s}=0}^{m_{1} k_{1}+\cdots+m_{s} k_{s} \leq n m_{1}^{\prime} k_{1}^{\prime}+\cdots+m_{t}^{\prime} k_{t}^{\prime} \leq n^{\prime}} \sum_{k_{1}^{\prime}, \ldots, k_{t}^{\prime}=0}(-n)_{m_{1} k_{1}+\cdots+m_{s} k_{s}}\left(-n^{\prime}\right)_{m_{1}^{\prime} k_{1}^{\prime}+\cdots+m_{t}^{\prime} k_{t}^{\prime}} \\
& \times A\left(n, k_{1}, \ldots, k_{s}\right) A^{\prime}\left(n^{\prime}, k_{1}^{\prime}, \ldots, k_{t}^{\prime}\right) \frac{a_{1}^{k_{1}}}{k_{1} !} \cdots \frac{a_{s}^{k_{s}}}{k_{s} !} \cdot \frac{b_{1}^{k_{1}^{\prime}}}{k_{1}^{\prime} !} \cdots \frac{b_{t}^{k_{t}^{\prime}}}{k_{t}^{\prime} !} \\
B(\theta)= & \alpha^{\sum_{i=1}^{s} v_{i} k_{i}+\sum_{j=1}^{t} v_{j}^{\prime} k_{j}^{\prime}-\ell} \beta^{\sum_{i=1}^{s} w_{i} k_{i}+\sum_{j=1}^{t} w_{j}^{\prime} k_{j}^{\prime}-\lambda}
\end{aligned}
$$




$$
\begin{aligned}
& I(\psi)=\sum_{k_{1}, \ldots, k_{s}=0}^{m_{1} k_{1}+\cdots+m_{s} k_{s} \leq n}(-n)_{m_{1} k_{1}+\cdots+m_{s}} k_{s} \frac{\prod_{i=1}^{E}\left(e_{i}\right)_{k_{1} y_{i}^{\prime}+\cdots+k_{s} y_{i}^{(s)}}}{\prod_{i=1}^{G}\left(f_{i}\right)_{k_{1} \xi_{i}^{\prime}+\cdots+k_{s} \xi_{i}^{(s)}}} \\
& \times \frac{\prod_{i=1}^{U^{\prime}}\left(T_{i}^{\prime}\right)_{k_{1} p_{i}^{\prime}} \cdots \prod_{i=1}^{U^{(s)}}\left(T_{i}^{s}\right)_{k_{s} p_{i}^{(s)}}}{V^{(s)}} \cdot \frac{a_{1}^{k_{1}}}{k_{1} !} \cdots \frac{a_{s}^{k_{s}}}{k_{s} !} \\
& \prod_{i=1}\left(\Omega_{i}^{\prime}\right)_{k_{1} q_{i}^{\prime}} \cdots \prod_{i=1}\left(\Omega_{i}^{(s)}\right)_{k_{s} q_{i}^{(s)}} \\
& I^{*}\left(\psi^{*}\right)=\sum_{k_{1}^{\prime}, \ldots, k_{t}^{\prime}=0}^{m_{1} k_{1}^{\prime}+\cdots+m_{t} k_{t}^{\prime} \leq n^{\prime}}\left(-n^{\prime}\right)_{m_{1}^{\prime} k_{1}^{\prime}+\cdots+m_{t}^{\prime} k_{t}^{\prime}} \frac{\prod_{j=1}^{E^{\prime}}\left(e_{j}^{\prime}\right)_{k_{1}^{\prime} y_{j}^{\prime}+\cdots+k_{t}^{\prime} y_{j}^{(t)}}}{\prod_{j=1}^{G^{\prime}}\left(f_{j}^{\prime}\right)_{k_{1}^{\prime} \xi_{j}^{\prime}+\cdots+k_{t}^{\prime} \xi_{j}^{(t)}}} \\
& \times \frac{\prod_{i=1}^{\left(U^{*}\right)^{\prime}}\left(T_{j}^{\prime}\right)_{k_{1}^{\prime} p_{j}^{\prime}} \cdots \prod_{i=1}^{\left(U^{*}\right)^{t}}\left(T_{j}^{t}\right)_{k_{t}^{\prime} p_{j}^{t}}}{\prod_{i=1}^{\left(V^{*}\right)^{\prime}}\left(\Omega_{j}^{\prime}\right)_{k_{1}^{\prime} q_{j}^{\prime}} \cdots \prod_{i=1}^{\left(V^{*}\right)^{t}}\left(\Omega_{j}^{t}\right)_{k_{t}^{\prime} q_{j}^{t}}} \cdot \frac{b_{1}^{k_{1}^{\prime}}}{k_{1}^{\prime} !} \cdots \frac{b_{t}^{k_{t}^{\prime}}}{k_{t}^{\prime} !} .
\end{aligned}
$$

\section{Fractional integral formulae for the multivariable $H$-function}

We shall prove the fractional integral formulae

(a)

$$
\begin{aligned}
& { }_{c} I_{x}^{v}\left\{x^{\rho}(x+\alpha)^{\sigma}(x+\beta)^{\mu} S_{n}^{m_{1}, \ldots, m_{s}}\left[a_{1} x^{\mu_{1}}(x+\alpha)^{v_{1}}(x+\beta)^{w_{1}}, \ldots, a_{s} x^{u_{s}}(x+\alpha)^{v_{s}}(x+\beta)^{w_{s}}\right]\right. \\
& \times S_{n^{\prime}}^{m_{1}^{\prime}, \ldots, m_{t}^{\prime}}\left[b_{1} x^{u_{1}^{\prime}}(x+\alpha)^{v_{1}^{\prime}}(x+\beta)^{w_{1}^{\prime}}, \ldots, b_{t} x^{u_{t}^{\prime}}(x+\alpha)^{v_{t}^{\prime}}(x+\beta)^{w_{t}^{\prime}}\right] \\
& \left.\times H\left[z_{1} x^{u_{1}^{\prime \prime}}(x+\alpha)^{v_{1}^{\prime \prime}}(x+\beta)^{w_{1}^{\prime \prime}}, \ldots, z_{r} x^{u_{r}^{\prime \prime}}(x+\alpha)^{v_{r}^{\prime \prime}}(x+\beta)^{w_{r}^{\prime \prime}}\right]\right\} \\
& =\alpha^{\sigma} \beta^{\mu} x^{\rho} \sum_{\eta, \ell, \lambda=0}^{\infty} A(\phi) B(\theta) \frac{(-1)^{\eta}(x-c)^{\eta+v} x^{\sum_{i=1}^{s} u_{i} k_{i}+\sum_{j=1}^{t} u_{j}^{\prime} k_{j}^{\prime}+\ell+\lambda-\eta}}{\ell ! \lambda ! \Gamma v(\eta+v) \eta !} \\
& \times H_{p+3, Q+3:\left[P^{\prime}, Q^{\prime}\right] ; \cdots ;\left[P^{(r)}, Q^{(r)}\right]}^{\left.0, N+3:\left[M^{\prime}, N^{\prime}\right] ; \cdots ; M^{(r)}, N^{(r)}\right]}\left[\begin{array}{c|c}
z_{1} \alpha^{v_{1}^{\prime \prime}} \beta^{w_{1}^{\prime \prime}} x_{1}^{u_{1}^{\prime \prime}} & \left(-\rho-\sum_{i=1}^{s} u_{i} k_{i}-\sum_{j=1}^{t} u_{j}^{\prime} k_{j}^{\prime}-\ell-\lambda: u_{1}^{\prime \prime}, \ldots, u_{r}^{\prime \prime}\right), \\
\vdots & \\
z_{r} \alpha^{v_{r}^{\prime \prime}} \beta^{w_{r}^{\prime \prime}} x_{r}^{u_{r}^{\prime \prime}} & \left(\eta-\rho-\sum_{i=1}^{s} u_{i} k_{i}-\sum_{j=1}^{t} u_{j}^{\prime} k_{j}^{\prime}-\ell-\lambda: u_{1}^{\prime \prime}, \ldots, u_{r}^{\prime \prime}\right),
\end{array}\right.
\end{aligned}
$$




$$
\begin{aligned}
& \left(-\sigma-\sum_{i=1}^{s} v_{i} k_{i}-\sum_{j=1}^{t} v_{j}^{\prime} k_{j}^{\prime}: v_{1}^{\prime \prime}, \ldots, v_{r}^{\prime \prime}\right),\left(-\mu-\sum_{i=1}^{s} w_{i} k_{i}-\sum_{j=1}^{t} w_{j}^{\prime} k_{j}^{\prime}: w_{1}^{\prime \prime}, \ldots, w_{r}^{\prime \prime}\right), \\
& \left(\ell-\sigma-\sum_{i=1}^{s} v_{i} k_{i}-\sum_{j=1}^{t} v_{j}^{\prime} k_{j}^{\prime}: v_{1}^{\prime \prime}, \ldots, v_{r}^{\prime \prime}\right),\left(\lambda-\mu-\sum_{i=1}^{s} w_{i} k_{i}-\sum_{j=1}^{t} w_{j}^{\prime} k_{j}^{\prime}: w_{1}^{\prime \prime}, \ldots, w_{r}^{\prime \prime}\right), \\
& \left.\begin{array}{l}
{\left[(a): \theta^{\prime}, \ldots, \theta^{(r)}\right]:\left[b^{\prime}: \phi^{\prime}\right] ; \ldots ;\left[b^{(r)}: \phi^{(r)}\right]} \\
{\left[(c): \psi^{\prime} ; \ldots ; \psi^{(r)}\right]:\left[d^{\prime}: \delta^{\prime}\right] ; \ldots ;\left[d^{(r)}: \delta^{(r)}\right]}
\end{array}\right] .
\end{aligned}
$$

where

(i) $\Lambda_{i}>0, \min \left\{u_{i}, v_{i}, w_{i}, u_{j}^{\prime}, v_{j}^{\prime}, w_{j}^{\prime}, u_{i^{\prime}}^{\prime \prime}, v_{i^{\prime}}^{\prime \prime}, w_{i^{\prime}}^{\prime \prime}\right\}>0, i=1, \ldots, s ; j=1, \ldots, t$; $i^{\prime}=1, \ldots, r$

(ii) $\left|\arg \left(z_{i}\right)\right|<\frac{\Lambda_{i} \pi}{2}$,

(iii) $\operatorname{Re}(\rho)+\sum_{i=1}^{r} \alpha_{i} u_{i}^{\prime \prime}>-1$ and $m_{i}$ and $m_{i}^{\prime}$ are arbitrary positive integers.

Result (2.1) can also be written in the following form:

$$
\begin{aligned}
& I_{x}^{v}\left\{x^{\rho}(x+\alpha)^{\sigma}(x+\beta)^{\mu} S_{n}^{m_{1}, \ldots, m_{s}}\left[a_{1} x^{u_{1}}(x+\alpha)^{v_{1}}(x+\beta)^{w_{1}}, \ldots, a_{s} x^{u_{s}}(x+\alpha)^{v_{s}}(x+\beta)^{w_{s}}\right]\right. \\
& \times S_{n^{\prime}}^{m_{1}^{\prime}, \ldots, m_{t}^{\prime}}\left[b_{1} x^{u_{1}^{\prime}}(x+\alpha)^{v_{1}^{\prime}}(x+\beta)^{w_{1}^{\prime}}, \ldots, b_{t} x^{u_{t}^{\prime}}(x+\alpha)^{v_{t}^{\prime}}(x+\beta)^{w_{t}^{\prime}}\right] \\
& \left.\times H\left[z_{1} x^{u_{1}^{\prime \prime}}(x+\alpha)^{v_{1}^{\prime \prime}}(x+\beta)^{w_{1}^{\prime \prime}}, \ldots, z_{r} x^{u_{r}^{\prime \prime}}(x+\alpha)^{v_{r}^{\prime \prime}}(x+\beta)^{w_{r}^{\prime \prime}}\right]\right\} \\
& =\alpha^{\sigma} \beta^{\mu} x^{\rho+v} \sum_{\ell, \lambda=0}^{\infty} A(\phi) B(\theta) \frac{\sum_{i=1}^{s} u_{i} k_{i}+\sum_{j=1}^{t} u_{j}^{\prime} k_{j}^{\prime}+\ell+\lambda}{\ell ! \lambda !} \\
& \times H_{p+3, Q+3:\left[P^{\prime}, Q^{\prime}\right] ; \ldots ;\left[P^{(r)}, Q^{(r)}\right]}^{0, N+3:\left[M^{\prime}, N^{\prime} ; \ldots ;\left[M^{(r)}, N^{(r)}\right]\right.}\left[\begin{array}{c|c}
z_{1} \alpha^{v_{1}^{\prime \prime}} \beta^{w_{1}^{\prime \prime}} x_{1}^{u_{1}^{\prime \prime}} & \left(-\rho-\sum_{i=1}^{s} u_{i} k_{i}-\sum_{j=1}^{t} u_{j}^{\prime} k_{j}^{\prime}-\ell-\lambda: u_{1}^{\prime \prime}, \ldots, u_{r}^{\prime \prime}\right), \\
\vdots & \\
z_{r} \alpha_{r}^{v_{r}^{\prime \prime}} \beta_{r}^{w_{r}^{\prime \prime}} x_{r}^{u_{r}^{\prime \prime}} & \left(-v-\rho-\sum_{i=1}^{s} u_{i} k_{i}-\sum_{j=1}^{t} u_{j}^{\prime} k_{j}^{\prime}-\ell-\lambda: u_{1}^{\prime \prime}, \ldots, u_{r}^{\prime \prime}\right),
\end{array}\right. \\
& \left(-\sigma-\sum_{i=1}^{s} v_{i} k_{i}-\sum_{j=1}^{t} v_{j}^{\prime} k_{j}^{\prime}: v_{1}^{\prime \prime}, \ldots, v_{r}^{\prime \prime}\right),\left(-\mu-\sum_{i=1}^{s} w_{i} k_{i}-\sum_{j=1}^{t} w_{j}^{\prime} k_{j}^{\prime}: w_{1}^{\prime \prime}, \ldots, w_{r}^{\prime \prime}\right), \\
& \left(\ell-\sigma-\sum_{i=1}^{s} v_{i} k_{i}-\sum_{j=1}^{t} v_{j}^{\prime} k_{j}^{\prime}: v_{1}^{\prime \prime}, \ldots, v_{r}^{\prime \prime}\right),\left(\lambda-\mu-\sum_{i=1}^{s} w_{i} k_{i}-\sum_{j=1}^{t} w_{j}^{\prime} k_{j}^{\prime}: w_{1}^{\prime \prime}, \ldots, w_{r}^{\prime \prime}\right), \\
& \left.\begin{array}{l}
{\left[(a): \theta^{\prime}, \ldots, \theta^{(r)}\right]:\left[b^{\prime}: \phi^{\prime}\right] ; \ldots ;\left[b^{(r)}: \phi^{(r)}\right]} \\
{\left[(c): \psi^{\prime} ; \ldots ; \psi^{(r)}\right]:\left[d^{\prime}: \delta^{\prime}\right] ; \ldots ;\left[d^{(r)}: \delta^{(r)}\right]}
\end{array}\right] .
\end{aligned}
$$

(b)

$$
\begin{aligned}
I_{x}^{\gamma, v} & \left\{x^{\rho}(x+\alpha)^{\sigma}(x+\beta)^{\mu} S_{n}^{m_{1}, \ldots, m_{s}}\left[a_{1} x^{u_{1}}(x+\alpha)^{v_{1}}(x+\beta)^{w_{1}}, \ldots, a_{s} x^{u_{s}}(x+\alpha)^{v_{s}}(x+\beta)^{w_{s}}\right]\right. \\
& \times S_{n^{\prime}}^{m_{1}^{\prime}, \ldots, m_{t}^{\prime}}\left[b_{1} x^{u_{1}^{\prime}}(x+\alpha)^{v_{1}^{\prime}}(x+\beta)^{w_{1}^{\prime}}, \ldots, b_{t} x^{u_{t}^{\prime}}(x+\alpha)^{v_{t}^{\prime}}(x+\beta)^{w_{t}^{\prime}}\right] \\
& \left.\times H\left[z_{1} x^{u_{1}^{\prime \prime}}(x+\alpha)^{v_{1}^{\prime \prime}}(x+\beta)^{w_{1}^{\prime \prime}}, \ldots, z_{r} x^{u_{r}^{\prime \prime}}(x+\alpha)^{v_{r}^{\prime \prime}}(x+\beta)^{w_{r}^{\prime \prime}}\right]\right\}
\end{aligned}
$$




$$
\begin{aligned}
& =\alpha^{\sigma} \beta^{\mu} x^{\rho} \sum_{\ell, \lambda=0}^{\infty} A(\phi) B(\theta) \frac{\sum_{i=1}^{s} u_{i} k_{i}+\sum_{j=1}^{t} u_{j}^{\prime} k_{j}^{\prime}+\ell+\lambda}{\ell ! \lambda !}
\end{aligned}
$$

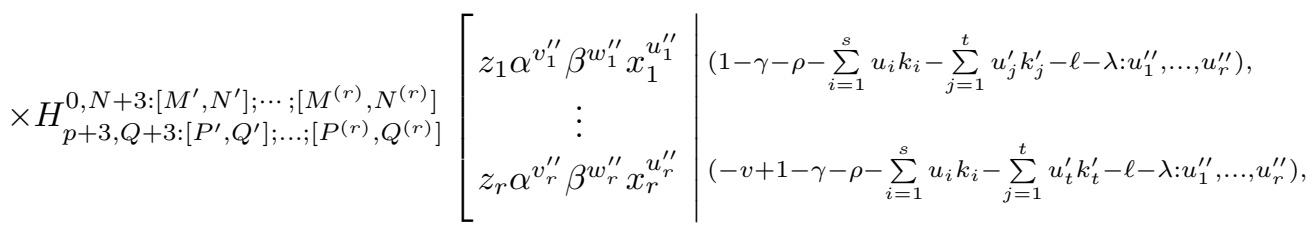

$$
\begin{aligned}
& \left(-\sigma-\sum_{i=1}^{s} v_{i} k_{i}-\sum_{j=1}^{t} v_{j}^{\prime} k_{j}^{\prime}: v_{1}^{\prime \prime}, \ldots, v_{r}^{\prime \prime}\right),\left(-\mu-\sum_{i=1}^{s} w_{i} k_{i}-\sum_{j=1}^{t} w_{j}^{\prime} k_{j}^{\prime}: w_{1}^{\prime \prime}, \ldots, w_{r}^{\prime \prime}\right), \\
& \left(\ell-\sigma-\sum_{i=1}^{s} v_{i} k_{i}-\sum_{j=1}^{t} v_{j}^{\prime} k_{j}^{\prime}: v_{1}^{\prime \prime}, \ldots, v_{r}^{\prime \prime}\right),\left(\lambda-\mu-\sum_{i=1}^{s} w_{i} k_{i}-\sum_{j=1}^{t} w_{j}^{\prime} k_{j}^{\prime}: w_{1}^{\prime \prime}, \ldots, w_{r}^{\prime \prime}\right), \\
& \left.\begin{array}{l}
{\left[(a): \theta^{\prime}, \ldots, \theta^{(r)}\right]:\left[b^{\prime}: \phi^{\prime}\right] ; \ldots ;\left[b^{(r)}: \phi^{(r)}\right]} \\
{\left[(c): \psi^{\prime} ; \ldots ; \psi^{(r)}\right]:\left[d^{\prime}: \delta^{\prime}\right] ; \ldots ;\left[d^{(r)}: \delta^{(r)}\right]}
\end{array}\right] .
\end{aligned}
$$

where

(i) $\Lambda_{i}>0, \min \left\{u_{i}, v_{i}, w_{i}, u_{j}^{\prime}, v_{j}^{\prime}, w_{j}^{\prime}, u_{i^{\prime}}^{\prime \prime}, v_{i^{\prime}}^{\prime \prime}, w_{i^{\prime}}^{\prime \prime}\right\}>0, i=1, \ldots, s ; j=1, \ldots, t$; $i^{\prime}=1, \ldots, r$

(ii) $\left|\arg \left(z_{i}\right)\right|<\frac{\Lambda_{i} \pi}{2}$,

(iii) $\operatorname{Re}(\rho)+\sum_{i=1}^{r} \alpha_{i} u_{i}^{\prime \prime}>-\eta$ and $m_{i}$ and $m_{i}^{\prime}$ are arbitrary positive integers.

Proof. In order to prove (2.1), we first replace the multivariable $H$-function occurring on the left hand side by its Mellin-Barnes contour integral form given by (1.1) and express both general class of multivariable polynomials in series form given by (1.5). Now we collect the powers of $x,(x+\alpha)$ and $(x+\beta)$ and apply the binomial expansion

$$
(x+\alpha)^{\sigma}=\alpha^{\sigma} \sum_{\ell=0}^{\infty}\left(\begin{array}{l}
\sigma \\
\ell
\end{array}\right)\left(\frac{x}{\alpha}\right)^{\ell} \quad(|x / \alpha|<1),
$$

then we apply the fractional integral formula [2]

$$
{ }_{c} I_{x}^{v}\left\{x^{g}\right\}=\sum_{\eta=0}^{\infty} \frac{(-1)^{\eta}(x-c)^{\eta+v}}{\Gamma v(\eta+v) \eta !} \cdot \frac{\Gamma g+1}{\Gamma g-\eta+1} x^{g-\eta} \quad[\operatorname{Re}(g)>-1],
$$

and interpret the resulting Mellin-Barnes contour integral as an $H$-function of $r$-variables which is permissible under the stated conditions. We are thus led finally to the fractional integral formula (2.1). 
By the application of the following formula

$$
\begin{aligned}
& \sum_{\eta=0}^{\infty} \frac{(-1)^{\eta}}{\Gamma v(v+\eta) \eta !} H_{P, Q+1:\left[P^{\prime}, Q^{\prime}\right] ; \ldots ;\left[P^{(r)}, Q^{(r)}\right]}^{0, N:\left[M^{\prime}, N^{\prime}\right] ; \ldots ;\left[M^{(r)}, N^{(r)}\right]}\left[\begin{array}{c|cc}
x_{1} & {\left[(a): \theta^{\prime}, \ldots, \theta^{(r)}\right]:} & {\left[b^{\prime}: \phi^{\prime}\right] ; \ldots ;\left[b^{r}: \phi^{r}\right]} \\
\vdots & & \left(\eta-k: u_{1}, \ldots, u_{r}\right),\left[(c): \psi^{\prime}, \ldots, \psi^{(r)}\right]:\left[d^{\prime}: \delta^{\prime}\right] ; \ldots ;\left[d^{r}: \delta^{r}\right]
\end{array}\right] \\
& =H_{P, Q+1:\left[P^{\prime}, Q^{\prime}\right] ; \ldots ;\left[P^{(r)}, Q^{(r)}\right]}^{0, N:\left[M^{\prime}, N^{\prime}\right] \ldots ;\left[M^{(r)}, N^{(r)}\right]}\left[\begin{array}{c|lc}
x_{1} & {\left[(a): \theta^{\prime}, \ldots, \theta^{(r)}\right]:} & {\left[b^{\prime}: \phi^{\prime}\right] ; \ldots ;\left[b^{r}: \phi^{r}\right]} \\
\vdots & & \left(v-k: u_{1}, \ldots, u_{r}\right),\left[(c): \psi^{\prime}, \ldots, \psi^{(r)}\right]:\left[d^{\prime}: \delta^{\prime}\right] ; \ldots ;\left[d^{r}: \delta^{r}\right]
\end{array}\right] .
\end{aligned}
$$

We can evaluated result (2.2) which is an another form of result (2.1).

For result (2.3) we proceed on similar lines as adopted in result (2.1) and using

$$
I_{x}^{\gamma, v}\left\{x^{g}\right\}=\frac{\Gamma g+\gamma}{\Gamma g+\gamma+v} x^{g}, \quad \operatorname{Re}(g)>-\gamma .
$$

We can generate the result (2.3).

\section{Special cases}

Taking

$$
A\left(n ; k_{1}, \ldots, k_{s}\right)=\frac{\prod_{i=1}^{E}\left(e_{i}\right)_{k_{1} y_{i}^{\prime}+\cdots+k_{s} y_{i}^{(s)}}}{\prod_{i=1}^{G}\left(f_{i}\right)_{k_{1} \xi_{i}^{\prime}+\cdots+k_{s} \xi_{i}^{(s)}}} \cdot \frac{\prod_{i=1}^{U^{\prime}}\left(T_{i}^{\prime}\right)_{k_{1} p_{i}^{\prime}} \cdots \prod_{i=1}^{V^{\prime}}\left(T_{i}^{(s)}\right)_{k_{s} p_{i}^{(s)}}}{\prod_{i=1}^{V^{\prime}}\left(\Omega_{i}^{\prime}\right)_{k_{1} q_{i}^{\prime}} \cdots \prod_{i=1}^{V^{(s)}}\left(\Omega_{i}^{(s)}\right)_{k_{s} q_{i}^{s}}}
$$

and

$$
A^{\prime}\left(n^{\prime} ; k_{1}^{\prime}, \ldots, k_{t}^{\prime}\right)=\frac{\prod_{j=1}^{E^{\prime}}\left(e_{j}^{\prime}\right)_{k_{1}^{\prime} y_{j}^{\prime}+\cdots+k_{t}^{\prime} y_{j}^{(t)}}}{\prod_{j=1}^{G^{\prime}}\left(f_{j}^{\prime}\right)_{k_{1}^{\prime} \xi_{j}^{\prime}+\cdots+k_{t}^{\prime} \xi_{j}^{(t)}}} \cdot \frac{\prod_{j=1}^{\left(U^{*}\right)^{\prime}}\left(T_{j}^{\prime}\right)_{k_{1}^{\prime} p_{j}^{\prime}} \cdots \prod_{j=1}^{\left(U^{*}\right)^{t}}\left(T_{j}^{t}\right)_{k_{t}^{\prime} p_{j}^{t}}}{\prod_{j=1}^{\left(U^{*}\right.}\left(\Omega_{j}^{\prime}\right)_{k_{1}^{\prime} q_{j}^{\prime}} \cdots \prod_{j=1}^{\left(V^{*}\right)^{t}}\left(\Omega_{j}^{t}\right)_{k_{t}^{\prime} q_{j}^{t}}},
$$

in (1.5), $S_{n}^{m_{1}, \ldots, m_{s}}\left[w_{1}, \ldots, w_{s}\right]$ and $S_{n}^{m_{1}^{\prime}, \ldots, m_{t}^{\prime}}\left[w_{1}^{\prime}, \ldots, w_{t}^{\prime}\right]$ reduce to the generalized Lauricella function of Srivastava, H. M. and Daoust, M. C. [7. p.454] as follows

$$
\begin{aligned}
& S_{n}^{m_{1}, \ldots, m_{s}}\left[w_{1}, \ldots, w_{s}\right] \\
& =F_{G: V^{\prime} ; \ldots ; V^{(s)}}^{1+E: U^{\prime} ; \ldots ; U^{(s)}}\left[\begin{array}{c}
\left(-n: m_{1}, \ldots, m_{s}\right),\left((e): y^{\prime}, \ldots, y^{s}\right):\left(\left(T^{\prime}\right): p^{\prime}\right) ; \ldots ;\left(\left(T^{(s)}\right): p^{(s)}\right) ; \\
\left((f): \xi^{\prime}, \ldots, \xi^{s}\right):\left(\left(\Omega^{\prime}\right): q^{\prime}\right) ; \ldots ;\left(\left(\Omega^{s}\right): q^{s}\right) ;
\end{array} w_{1}, \ldots, w_{s}\right]
\end{aligned}
$$


and

$$
\begin{aligned}
& S_{n^{\prime}}^{m_{1}^{\prime}, \ldots, m_{t}^{\prime}}\left[w_{1}, \ldots, w_{t}\right] \\
& =F_{* G^{\prime}: V^{\prime} ; \ldots ; V^{t}}^{1+E^{\prime} ; \ldots ; V^{t}}\left[\begin{array}{c}
\left(-n^{\prime}: m_{1}^{\prime}, \ldots, m_{t}^{\prime}\right),\left(\left(e^{\prime}\right): y^{\prime}, \ldots, y^{t}\right):\left(\left(T^{\prime}\right): p^{\prime}\right) ; \ldots ;\left(\left(T^{(t)}\right): p^{(t)}\right) ; \\
\left((f): \xi^{\prime}, \ldots, \xi^{t}\right):\left(\left(\Omega^{(\prime)}\right): q^{(\prime)}\right) ; \ldots ;\left(\left(\Omega^{t}\right): q^{t}\right) ;
\end{array} w_{1}, \ldots, w_{s}\right]
\end{aligned}
$$

and the result (2.2) and (2.3) readily reduce to the result involving generalized Lauricella function. For example

1.

$$
\begin{aligned}
& I_{x}^{v}\left\{x^{\rho}(x+\alpha)^{\sigma}(x+\beta)^{\mu} F\left[a_{1} x^{u_{1}}(x+\alpha)^{v_{1}}(x+\beta)^{w_{1}}, \ldots, a_{s} x^{u_{s}}(x+\alpha)^{v_{s}}(x+\beta)^{w_{s}}\right]\right. \\
& \times F_{*}\left[b_{1} x^{u_{1}^{\prime}}(x+\alpha)^{v_{1}^{\prime}}(x+\beta)^{w_{1}^{\prime}}, \ldots, b_{t} x^{u_{t}^{\prime}}(x+\alpha)^{v_{t}^{\prime}}(x+\beta)^{w_{t}^{\prime}}\right] \\
& \left.\times H\left[z_{1} x^{u_{1}^{\prime \prime}}(x+\alpha)^{v_{1}^{\prime \prime}}(x+\beta)^{w_{1}^{\prime \prime}}, \ldots, z_{r} x^{u_{r}^{\prime \prime}}(x+\alpha)^{v_{r}^{\prime \prime}}(x+\beta)^{w_{r}^{\prime \prime}}\right]\right\} \\
& =\alpha^{\sigma} \beta^{\mu} x^{\rho+v} \sum_{\ell, \lambda=0}^{\infty} I^{*}\left(\psi^{*}\right) B(\theta) \frac{x^{\sum_{i=1}^{r} u_{i} k_{i}+\sum_{j=1}^{t} u_{j}^{\prime} k_{j}^{\prime}+\ell+\lambda}}{\ell ! \lambda !} \\
& \times H_{P+3, Q+3:\left[P^{\prime}, Q^{\prime}\right] ; \ldots ;\left[P^{(r)}, Q^{(r)}\right]}^{\left.0, N+3:\left[M^{\prime}, N^{\prime}\right] \ldots ; M^{(r)}, N^{(r)}\right]}\left[\begin{array}{c|c}
z_{1} \alpha^{v_{1}^{\prime \prime}} \beta^{w_{1}^{\prime \prime}} x_{1}^{u_{1}^{\prime \prime}} & \left(-\rho-\sum_{i=1}^{s} u_{i} k_{i}-\sum_{j=1}^{t} u_{j}^{\prime} k_{j}^{\prime}-\ell-\lambda: u_{1}^{\prime \prime}, \ldots, u_{r}^{\prime \prime}\right), \\
\vdots & \\
z_{r} \alpha_{r}^{v_{r}^{\prime \prime}} \beta^{w_{r}^{\prime \prime}} x_{r}^{u_{r}^{\prime \prime}} & \left(-v-\rho-\sum_{i=1}^{s} u_{i} k_{i}-\sum_{j=1}^{t} u_{j}^{\prime} k_{j}^{\prime}-\ell-\lambda: u_{1}^{\prime \prime}, \ldots, u_{r}^{\prime \prime}\right),
\end{array}\right. \\
& \left(-\sigma-\sum_{i=1}^{s} v_{i} k_{i}-\sum_{j=1}^{t} v_{j}^{\prime} k_{j}^{\prime}: v_{1}^{\prime \prime}, \ldots, v_{r}^{\prime \prime}\right),\left(-\mu-\sum_{i=1}^{s} w_{i} k_{i}-\sum_{j=1}^{t} w_{j}^{\prime} k_{j}^{\prime}: w_{1}^{\prime \prime}, \ldots, w_{r}^{\prime \prime}\right), \\
& \left(\ell-\sigma-\sum_{i=1}^{s} v_{i} k_{i}-\sum_{j=1}^{t} v_{j}^{\prime} k_{j}^{\prime}: v_{1}^{\prime \prime}, \ldots, v_{r}^{\prime \prime}\right),\left(\lambda-\mu-\sum_{i=1}^{s} w_{i} k_{i}-\sum_{j=1}^{t} w_{j}^{\prime} k_{j}^{\prime}: w_{1}^{\prime \prime}, \ldots, w_{r}^{\prime \prime}\right), \\
& \left.\begin{array}{l}
{\left[(a): \theta^{\prime}, \ldots, \theta^{(r)}\right]:\left[b^{\prime}: \phi^{\prime}\right] ; \ldots ;\left[b^{(r)}: \phi^{(r)}\right]} \\
{\left[(c): \psi^{\prime} ; \ldots ; \psi^{(r)}\right]:\left[d^{\prime}: \delta^{\prime}\right] ; \ldots ;\left[d^{(r)}: \delta^{(r)}\right]}
\end{array}\right] .
\end{aligned}
$$

2.

$$
\begin{aligned}
I_{x}^{\gamma, v}\{ & x^{\rho}(x+\alpha)^{\sigma}(x+\beta)^{\mu} F\left[a_{1} x^{u_{1}}(x+\alpha)^{v_{1}}(x+\beta)^{w_{1}}, \ldots, a_{s} x^{u_{s}}(x+\alpha)^{v_{s}}(x+\beta)^{w_{s}}\right] \\
& \times F_{*}\left[b_{1} x^{u_{1}^{\prime}}(x+\alpha)^{v_{1}^{\prime}}(x+\beta)^{w_{1}^{\prime}}, \ldots, b_{t} x^{u_{t}^{\prime}}(x+\alpha)^{v_{t}^{\prime}}(x+\beta)^{w_{t}^{\prime}}\right] \\
& \left.\times H\left[z_{1} x^{u_{1}^{\prime \prime}}(x+\alpha)^{v_{1}^{\prime \prime}}(x+\beta)^{w_{1}^{\prime \prime}}, \ldots, z_{r} x^{u_{r}^{\prime \prime}}(x+\alpha)^{v_{r}^{\prime \prime}}(x+\beta)^{w_{r}^{\prime \prime}}\right]\right\} \\
= & \alpha^{\sigma} \beta^{\mu} x^{\rho} \sum_{\ell, \lambda=0}^{\infty} I(\psi) I^{*}\left(\psi^{*}\right) B(\theta) \frac{\sum_{i=1}^{\sum_{i=1}^{r} u_{i} k_{i}+\sum_{j=1}^{t} u_{j}^{\prime} k_{j}^{\prime}+\ell+\lambda}}{\ell ! \lambda !}
\end{aligned}
$$




$$
\begin{aligned}
& \times H_{P+3, Q+3:\left[P^{\prime}, Q^{\prime}\right] ; \ldots ;\left[P^{(r)}, Q^{(r)}\right]}^{0, N+3:\left[M^{\prime}, N^{\prime}\right] ;\left[M^{(r)}, N^{(r)}\right]}\left[\begin{array}{c|c}
z_{1} \alpha^{v_{1}^{\prime \prime}} \beta^{w_{1}^{\prime \prime}} x_{1}^{u_{1}^{\prime \prime}} & \left(1-\gamma-\rho-\sum_{i=1}^{s} u_{i} k_{i}-\sum_{j=1}^{t} u_{j}^{\prime} k_{j}^{\prime}-\ell-\lambda: u_{1}^{\prime \prime}, \ldots, u_{r}^{\prime \prime}\right), \\
\vdots & \\
z_{r} \alpha^{v_{r}^{\prime \prime}} \beta^{w_{r}^{\prime \prime}} x_{r}^{u_{r}^{\prime \prime}} & \left(-v+1-\gamma-\rho-\sum_{i=1}^{s} u_{i} k_{i}-\sum_{j=1}^{t} u_{j}^{\prime} k_{j}^{\prime}-\ell-\lambda: u_{1}^{\prime \prime}, \ldots, u_{r}^{\prime \prime}\right),
\end{array}\right. \\
& \left(-\sigma-\sum_{i=1}^{s} v_{i} k_{i}-\sum_{j=1}^{t} v_{j}^{\prime} k_{j}^{\prime}: v_{1}^{\prime \prime}, \ldots, v_{r}^{\prime \prime}\right),\left(-\mu-\sum_{i=1}^{s} w_{i} k_{i}-\sum_{j=1}^{t} w_{j}^{\prime} k_{j}^{\prime}: w_{1}^{\prime \prime}, \ldots, w_{r}^{\prime \prime}\right), \\
& \left(\ell-\sigma-\sum_{i=1}^{s} v_{i} k_{i}-\sum_{j=1}^{t} v_{j}^{\prime} k_{j}^{\prime}: v_{1}^{\prime \prime}, \ldots, v_{r}^{\prime \prime}\right),\left(\lambda-\mu-\sum_{i=1}^{s} w_{i} k_{i}-\sum_{j=1}^{t} w_{j}^{\prime} k_{j}^{\prime}: w_{1}^{\prime \prime}, \ldots, w_{r}^{\prime \prime}\right), \\
& \left.\begin{array}{l}
{\left[(a): \theta^{\prime}, \ldots, \theta^{(r)}\right]:\left[b^{\prime}: \phi^{\prime}\right] ; \ldots ;\left[b^{(r)}: \phi^{(r)}\right]} \\
{\left[(c): \psi^{\prime} ; \ldots ; \psi^{(r)}\right]:\left[d^{\prime}: \delta^{\prime}\right] ; \ldots ;\left[d^{(r)}: \delta^{(r)}\right]}
\end{array}\right] .
\end{aligned}
$$

3. By setting $s=t=1$ in (2.1) through (2.3), we arrive at the results [15] after a little simplification.

4. By putting $s=t=1$ and then $\mu=0, w_{i}=0(i=1, \ldots, r), m_{1}^{\prime}, n^{\prime}=0$ in integral formula (2.3), we arrive at the results obtained by Gupta and Agrawal [14].

5. If we put $\mu=0, n=n^{\prime}=0$ and $w_{i}=0(i=1, \ldots, r)$ in (2.2), we get a result which is the same as obtained by Srivastava et al. 12].

6. Taking $m^{\prime}=n^{\prime}=k^{\prime}(i=1, \ldots, t)=0$ and $w_{i}=0(i=1, \ldots, r)$ and $s=1$ in equation (2.1), we get another result of Gupta et al. 14].

\section{Acknowledgement}

The authors are grateful to Professor H. M. Srivastava, University of Victoria, Canada for his kind help and valuable suggestions in the preparation of this paper and also to the worthy referee for fruitful suggestions.

\section{References}

[1] A. A. Kilbas and M. Saigo, Fractional calculus of the $H$-function, Fukuoka Univ. Sci. rep. 28(1998), 41-51.

[2] B. Ross, Fractional calculus and its applications, Lecture notes in math. Vol.457, SpringerVerlag, New York, 1975.

[3] H. L. Manocha and B. L. Sharma, Fractional derivatives and summation, J. Indian Math. Soc. (N. S.) 38(1974), 371-382.

[4] H. M. Srivastava, A contour integral involving Fox's H-function, Indian J. Math. 14(1972), 1-6.

[5] H. M. Srivastava, K. C. Gupta and S. P. Goyal, The $H$-Functions of One and Two Variables with Applications, South Asian publishers, New Delhi, 1982.

[6] H. M. Srivastava and M. A. Hussain, Fractional integration of the $H$-function of several variables, Comput. Math. Appl. 30(1995), 73-85. 
[7] H. M. Srivastava and M. C. Daoust, Certain generalized Neumann expansions associated with the Kampé de Fériet function, Nederl. Akad. Wetensch. Indag. Math. 31(1969), 449457.

[8] H. M. Srivastava and M. Garg, Some integrals involving a general class of polynomials and the multivariable H-function, Rev. Roumaine Phys. 32(1987), 685-692.

[9] H. M. Srivastava and N. P. Singh, The integration of certain products of the multivariable $H$-function with a general class of polynomials, Rend, Circ. Mat. Palermo Ser. II 32(1983), 157-187.

[10] H. M. Srivastava, R. C. Singh Chandel and P. K. Vishwakarma, Fractional derivatives of certain generalized hypergeometric functions of several variables, J. Math. Anal. Appl. 184(1994), 560-572.

[11] H. M. Srivastava and R. Panda, Some bilateral generating functions for a class of generalized hypergeometric polynomials, J. Reine Angew. Math. 283/284(1976), 265-274.

[12] H. M. Srivastava and S. P. Goyal, Fractional derivatives of the $H$-function of several variables, J. Math. Anal. Appl. 112(1985), 641-651.

[13] K. B. Oldham and J. Spanier, The Fractional Calculus, Academic Press, New York/London, 1974.

[14] K. C. Gupta and S. M. Agrawal, Fractional integral formulae involving a general class of polynomials and the multivariable H-functions, Proc. Indian Acad. Sci. (Math. Sci.) 99(1989), 169-173.

[15] H. S. Dhami and M. K. Gaira, Fractional integral formulae involving the product of a general class of polynomials and the multivariable $H$-function, IMA, Univ. of Minnesota, U.S.A., 1999.

[16] M.-P. Chen, H. M. Srivastava and C.-S. Yu, Some operators of fractional calculus and their applications involving a new class of analytic function, Appl. Math. Comput. 91(1998), 285-296.

[17] M. Saigo and R. K. Raina, Fractional calculus operators associated with a general class of polynomials, Fukuoka Univ. Sci. Reports 18(1988), 15-22.

[18] R. C. Soni and D. Singh, Certain fractional derivatives formula involving the product of a general class of polynomials and the multivariable $H$-function, Proc. Indian Acad. Sci. (Math. Sci.) 122(2002), 551-562.

[19] S.-D. Lin, S.-T. Tu and H. M. Srivastava, New generating function for a class of generalized Hermite polynomials, J. Math. Anal. Appl. 261(2001), 479-496.

[20] V. B. L. Chaurasia and Neeti Gupta, General fractional integral operator, general class of polynomials and Fox's H-function, Soochow Journal of Mathematics 25(1999), 333-339.

[21] V. B. L. Chaurasia and Anju Godika, Fractional derivatives of certain special functions, Tamkang Journal of Mathematics 32 (2001), 103-109.

Department of Mathematics, University of Rajasthan, Jaipur-302004, India.

Department of Mathematics, Poornima College of Engineering, Jaipur-302022, India.

E-mail: harisingh.P@rediffmail.com 